\title{
Reactivity and selectivity in aggregate systems
}

\author{
Umberto Tonellato \\ University of Padova, Department of Organic Chemistry and Centro CNR \\ Meccanismi di Reazioni Organiche, via Marzolo, 1, 1-35131, Padova, Italy
}

\begin{abstract}
The issue of reactivity and selectivity in aggregates, mainly micelles or vesicles, is here shortly overviewed. The discussion is particularly addressed to reactivity, mainly in aqueous solutions, containing micelles and vesicles made of or containing inert, functionalized, and metal ion complexed surfactant-like substances, from a mechanistic point of view. Selectivity is mainly restricted to enantioselectivity effects in metalloaggregates of chiral ligand with transition metal ions and to the factors wich determine or influence such effects.
\end{abstract}

\section{Introduction.}

At the beginning of this century, the very same idea of aggregates, as intended today when reference is made to micelles, vesicles single- double-layers, etc., was a stranger to the scientific community. It was first brought to light (1a) seven decades ago to explain the fact that surfactants behave strangely in water. In spite of the interest of chemists for their applications, and that of biochemists addressed to the understanding of the role of membranes in biological systems, the world of aggregates remained largely undisclosed up to approximately forty years ago when a fast growing worldwide interest originated from scientists with diverse specialties: organic chemistry, physical chemistry, biochemistry, pharmaceutical chemistry, polymer chemistry. Such a widespread interest uncovered a large variety of aggregate structures so that, nowaday, it is difficult just to name all the types of morphologically different assemblies. While it is well understood that any relatively large organic structure dispersible in water, whatever its shape and resemblance to the classical structure of surfactants, may form its own aggregate species above the socalled critical micelle concentration $(\mathrm{cmc})$, or more generally, the critical aggregate concentration $(\mathrm{cac})$. The aggregation behavior is not a sole right of aqueous solutions: micelles are formed in other solvents, such as formamide. Microemulsions are the result of a delicate balance mediated by surfactants of oil and water microdomains, inverted micelles, and similar systems are formed in apolar solvents (2). In the last years, many aspects of surfactant chemistry have been influenced by those of supramolecular chemistry (3) which has grown and is still expanding so vigorously as to absorb and overshadow, at least in part, the interest for the chemistry of aggregates.

Physical organic chemists have always been in the forefront and their contribution to the development of this interdisciplinary branch of chemistry has been a major one. Since the early work by Hartley (1b) much interest was devoted to the fact that aqueous micelles can influence chemical rates and equilibria and such effects are observed for other self-assembling colloids. As the structure of the many aggregates and the properties of the solutions containing them were better defined, the reactivity of such media attracted much attention and the term micellar catalysis was coined and is still in use. Moreover, the studies of reactions in aggregates were stimulated by the fact that that they model, to a limited extent (see infra), reactions in biological assemblies and were included in the general area of the biomimetic chemistry.

The overall subject of reactivity in submicroscopic aggregates has expanded widely and to many different reactions. This paper is restricted mainly to the discussion of reactions in aqueous solutions containing micelles or vesicles from a mechanistic point of view and based on a choice of topics related mostly to our work and experience. As for the many aspects and physical properties of the aggregates, the

*Lecture presented at the 14th International Conference on Physical Organic Chemistry, Florianópolis, Brazil, 21-26 August 1998. Other presentations are published in this issue, pp. 1933-2040. 
reader is addressed to the many authoritative reviews of this subject $(2,4)$. Here, the attention will be focused on the reactivity and selectivity issue, with a short overview of: (i) the general principles that govern the effect of normal ("inert") aggregates; (ii) the reactivity of functionalized systems, including metalloaggregates; (iii) the selectivity observed in aggregates either made of or containing chiral amphiphiles.

\section{Reactivity}

\section{Inert aggregates. General principles.}

The key feature of any type of aggregate is that it incorporates solutes. Aggregates can bind neutral molecules or attract ions. By doing so, they affect rates and equilibria by bringing reactants together (or keeping them apart) as a result of concentration and "medium" effects. The kinetic effects (acceleration or retardation) much depend on the type of reaction, on the nature of the components of the aggregates, and on the reactants. As a widely accepted approach to quantitative treatments, the pseudophase model $(2,5)$ implies that reactivity is the sum of reactions occurring in the bulk solvent and the aggregate pseudophase, and a key point is the evaluation of the affinity of the solute for the aggregate. In the case of micelles, the best known aggregates are formed by inert surfactants here defined as amphiphilic molecular structures devoid of any covalently bound reactive functions. The model may be applied to unimolecular reactions (case A of Scheme 1) in a simple way. Assuming that the concentration of aggregated surfactant is that of total surfactant, $\mathrm{D}$, less that of monomer (given by the $c m c$ ), (i.e. $\left[\mathrm{D}_{\mathrm{M}}\right]=[\mathrm{D}]-\mathrm{cmc}$ ), the observed first order rate constant, $k_{1 \psi}$, is given by the equation shown in Scheme $1 A$, where $K_{A}=\left[A_{m}\right] /\left(\left[A_{w}\right]\left[D_{M}\right]\right)$ is the binding constant of $A$. The equation predicts saturation rate-[D] profiles such as that shown in the case of rate accelerations, and bears formal resemblance to that of Michaelis-Menten for enzyme kinetics so that $\mathrm{k}_{\mathrm{M}}$ and $\mathrm{K}_{\mathrm{A}}$ may be evaluated following the Lineweaver-Burks or similar equations, provided $\mathrm{k}_{\mathrm{w}}$ is known.

\section{A}

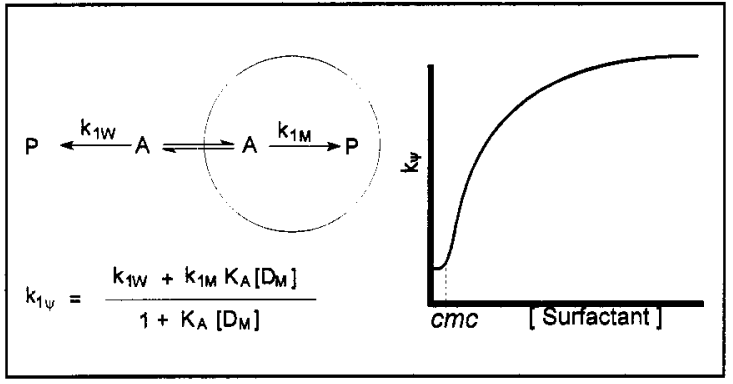

B

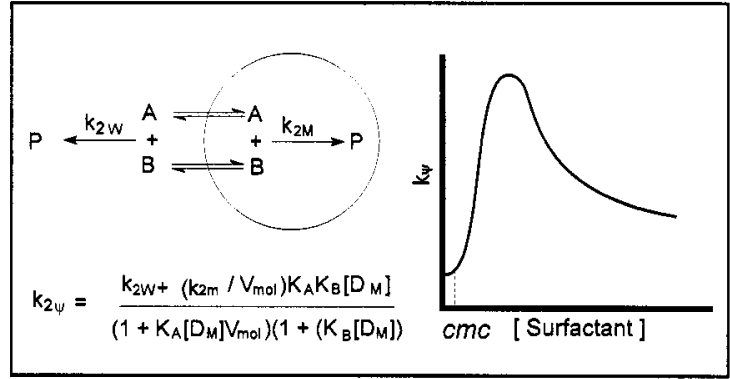

\section{Scheme 1}

In the case of a bimolecular reaction, $\mathrm{A}+\mathrm{B}$ (case $\mathrm{B}$ of Scheme 1), the observed second order rate constant $k_{2 \psi}$ is given by the equation shown in Scheme $1 \mathrm{~B}$, where $\mathrm{V}_{\mathrm{mol}}$ is the molar volume of the aggregated surfactant which can be regarded as the reaction volume. Such equation is a simplified form applied to dilute solutions, where $\left[D_{M}\right] V_{m o l} \ll<1$ and both reactants $A$ and $B$ bind rather strongly to micelles. Working in the presence of an excess of one of the partners (say B), from the observed pseudofirst order rate constant, $k_{\psi}$, the value of $k_{2 \psi}$ is given by the ratio $k_{\psi} /[B]$. These and similar equations agree with the observation that first order rate constants go through maxima with increasing surfactant concentration while keeping the overall reactant concentration constant. Addition of surfactant leads to binding of both surfactants in aggregates and hence to an increase in rate up to a point where further addition dilutes reactants in the micellar pseudophase and the rate decreases, as shown in the profile of Scheme 1B.

When at least one partner of the bimolecular reaction is an ionic species, the model is further complicated due to the problem of evaluating the effective concentration of ions at the reactive site of aggregates, when the competition of different ions (reactive and inert) in the interfacial region is taken into account. The pseudophase ion-exchange model, first developed by Romsted $(5 c, 6)$ and by Bunton and 
coworkers $(2,6)$, assumes that the micellar surface is a kind of ion exchanger but the total fractional occupation by counterions is constant whatever the nature and total concentration of these ions.

All treatments of raw kinetic data and supporting models are based on quite a number of assumption and approximations related to the complexity of the system. The large number of published papers on the subject leads to the following main indications: (i) The study of simple unimolecular reactions and the more staighforward treatment mainly indicate that aggregates behave as submicroscopic solvents and to a large extent they affect reaction rates according to known kinetic solvent effects. From the study of unimolecular water catalyzed processes (spontaneous hydrolysis of activated carboxylic esters) the reaction site appears to be the surface, a "wet" surface rather than the less polar interior. This led to a widely adopted assumption that the chemical ractions in micelles occur mainly in the so-called Stern layer, according to the Hartley model $(1 \mathrm{~b})$, which is approximately half the total volume of the spherical aggregate. (ii) In the case of bimolecular reactions, both medium and proximity effects are involved. A major problem in evaluating the second order rate constants in the aggregate pseudophase is related to the evaluation of the volume element of reaction which differs from one type of aggregate to another, and depends, also within the same type of aggregates, on the structure of reactants and reagents, and on the concentration and aggregation number of surfactants. Yet, on properly comparing the second order rate constants in water and in many aggregate pseudo-phases, the rate constants are surprisingly very similar. (iii) This generalization holds for reactions of nonionic reactants and also, upon treatment by the appropriate pseudophase ion exchange model, for the very common reactions involving one ionic reactant, notwithstanding the hydrophobicity of the aggregated substrate. Exceptions are restricted to few processes: in many of these cases (2b) the rate constant in the micellar pseudophase is smaller than in water but the difference may be accounted for on the basis of mechanistic considerations.

Thus, regardless of the method used, regardless of the type of aggregate (micelles, vesicles, microemulsions, layers, polyions), rate enhancements for bimolecular reactions appear to be mostly the result of concentrating the reagents on the aggregate surface and not on any peculiar property of the "organized" medium. However, an analysis of the different factors at play in reactions in association colloids cannot underrate the fact that aggregates may effectively alter reaction rates of processes involving lipophilic reactants under mild conditions.

\section{Functionalized aggregates.}

The study of bimolecular processes in micelles and a simple analysis of the equation in Scheme1B indicate that great advantage is expected if one of the reactants is not only in excess ([B]>>[A]) but also very strongly bound to the aggregate. Such idea, together with that of realizing systems that could mimic the action if not the efficacy of enzymes, particularly of hydrolases, led to the synthesis of functionalized surfactants and to the study of the reactivity of their micellar aggregates (7). Functionalized amphiphilic structures feature nucleophilic or basic groups covalently bound so that any functionalized surfactant is also a potential reactant for a scissile substrate. Many laboratories, including ours, devoted their studies to functionalized aggregates with typical reactive groups such as hydroxy, imidazole, amino, hydroxamic, hydroperoxy, most often inspired by the reactive sites of popular enzymes. As expected, very large rate enhancements were observed relative to reactions in water, employing dilute solutions of substrates (mostly activated esters), particularly in a $\mathrm{pH}$ interval when deprotonation of the functions occurs. In a series of functionalized cationic surfactants of general structure $\mathbf{1}$, the apparent $\mathrm{pKa}$ of the reactive function is lower by $0.5-1.5$ units than that of water dispersed (devoid of any paraffinic chain) analogs. Moreover, the deprotonation behavior in cationic assemblies is similar to that of polyelectrolytes so that the extent of acid dissociation of the solution is related to the apparent $\mathrm{pKa}$ by the modified Henderson equation which includes the degree of ionization (8). Likewise, the fraction of the dissociated nucleophilic species, $-\mathrm{F}^{-}$, in cationic aggregates at $\mathrm{pHs}<\mathrm{pKa}$ is larger than that in bulk solution. Intuitively, the rate equation that can be applied to functional micelles made of functionalized surfactants $D^{f}$ in reactions with a scissile substrate $S$ (under the conditions: $\left[D^{f}-c m c\right] \gg[S]$ ) is similar to equation $1 \mathrm{~A}$ and may be expressed in general terms as: $k_{1 \psi}=\left\{k_{W}+\left(k_{2 M} / V_{m o l}\right) K_{S}\left[D_{M}^{f}\right]\right\} /\left(1+K_{S}\left[D_{M}^{f}\right]\right)$ where $k_{1 \psi}$ is the observed first order rate constant, $k_{2 M}$ is the second order rate constant for the reaction in aggregate, and $\mathrm{K}_{\mathrm{S}}$ is the binding constant of the substrate to micelles. On increasing the concentration of $\mathrm{D}^{\mathrm{f}}$, saturation kinetics are observed. As mentioned above, the largest accelerations are observed for solutions at $\mathrm{pHs}$ lower but not too far from the $\mathrm{pKa}$ of the acidic function, where the effective nucleophilic species is $\mathrm{F}^{-}$. It was therefore of interest to evaluate the 
second order rate constant of the dissociated species, $k_{2 \mathrm{M}}^{-}$. Such a value can be estimated from the expression $\mathrm{k}_{2 \mathrm{M}}^{-}=\mathrm{k}_{2 \mathrm{M}}\left(1+\left[\mathrm{H}^{+}\right] / \mathrm{Ka}_{\mathrm{app}}\right)$. The $\mathrm{k}_{2 \mathrm{M}}^{-}$values were remarkably (and surprisingly) similar to the corresponding values observed for the corresponding reaction in water (8), considering all the approximations involved. Thus it may be concluded (although exceptions have been reported) that differential desolvation of anionic functions is not a factor, that the reaction site is substantially "wet", and that the kinetic benefits are due to the the high concentration of reactive functions experienced by the substrate once transferred into the small volume of the aggregate and, at least in a defined $\mathrm{pH}$ range, to changes in the acid dissociation equilibrium for essentially electrostatic reasons.

The $\mathrm{k}_{2 \mathrm{M}}^{-}$value was often defined as the catalytic rate constants mainly due to some analogies between functionalized micellar and enzymic reations. The analogy, however, is restricted to formal aspects and is, in general, unjustified since very little if any substrate selectivity or stereospecificity was observed. Moreover, except for a few cases, the turnover rate was too poor to justify the claim that functionalized aggregates are catalysts in their own right. As a matter of fact, micelles of the functionalized surfactants such as those of general structure 1 react with activated acyl derivatives in a two step process giving a fast acylation of the functions followed by a rather slow deacylation and regeneration of the "catalytic" species. It was only in the case of imidazole functionalized cationic surfactants, such as $\mathbf{1 b}$, that the turnover rate for the cleavage of $p$-nitrophenyl alkanoates was not far from those observed, for $\alpha$ chymotrypsin (6f) under comparable conditions. Large turnover rates were reported for gem-diol functionalized micelles which react rapidly with carboxylic or phosphoric acid ester to give an intermediate which spontaneously and rapidly decomposes to restore the catalytic functions (9).

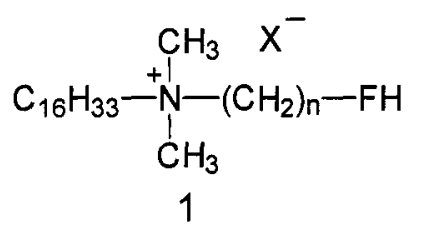<smiles>[X][N+](C)(CCO)CCCCCCC</smiles>

2 $F H=\underline{a}: O H(n=2) ; \underline{b}: m(n=1) ; \underline{c}: C O(P h)=N O H(n=1) ; x=C l, B r ; l m=N_{N H}$

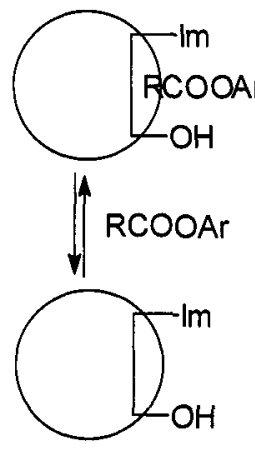

2

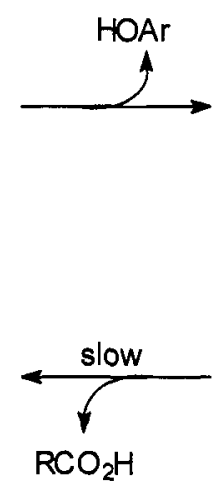

$\mathrm{RCO}_{2} \mathrm{H}$

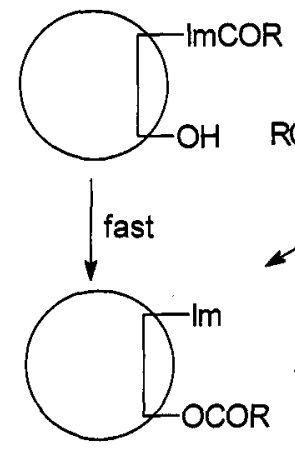

$2 a$

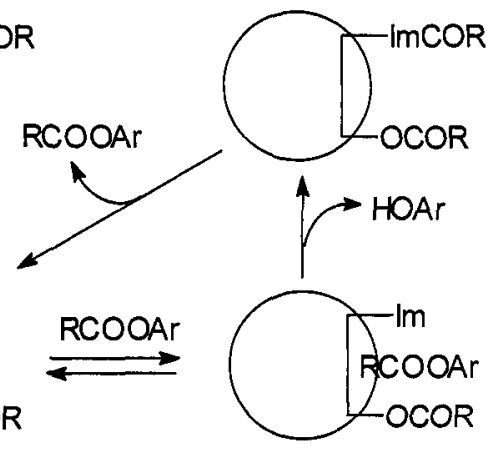

Scheme 2

Bifunctional surfactants were also explored as more refined biomimetic "catalysts". Imidazole and hydroxyl functionalized molecules such as 2 were investigated $(7 g, 8,11)$ hoping to achieve high reactivity and effective catalysis due to the presence of both nucleophilic and proton donor groups. The rate of cleavage of carboxylic acid esters (as well as the turnover rate measured using an excess substrate) was found similar to the that of the imidazole monofunctionalized aggregate, 1b. A relatively stable intermediate was the O-acylated derivative but a transient acylimidazole intermediate was also detected. The overall data point towards the stepwise mechanism illustrated in Scheme 2 and involving: (i) relatively fast acylation of the imidazole; (ii) fast acyl transfer to the $\mathrm{OH}$ function to give 2a that is slowly deacylated to water; (iii) when excess substrate is employed, intermediate $\mathbf{2 a}$ behaves as the monofunctionalized 
analog $1 \mathrm{~b}$, turning over at appreciable rate. Thus, no cooperation of the two functions is at play despite their proximity. Clearly, all function and water molecules being properly located,_enzymes can exploit a reaction site which is tailored for the transition state, at variance with the great looseness, mobility and wetness of the aggregate structure.

\section{In metalloaggregates.}

The term metalloaggregates was recently coined to indicate aggregates either made of or containing lipophilic metal ion complexes (12). Needless to say, the idea of investigating metalloaggregates was mainly inspired by the mode of action of metalloenzymes, notably the $\mathrm{Zn}(\mathrm{II})$ containing carboxypeptidase A. Other arguments, however, acted as a general stimulus to prepare effective catalysts for the hydrolysis of carboxylate and phosphate esters. The effect of (transition) metal ions may involve: (i) Lewis acid catalysis; (ii) charge neutralization; (iii) activation of nucleophilic functions or coordinated water molecules by decreasing its $\mathrm{pKa}$ and, hence, nucleophilic catalysis; (iv) assistance in leaving group departure. One of the advantages in using complexed metal ions is that they are less prone to form insoluble hydroxy complexes, and their inclusion in aggregates would add all the known and possibly new benefits. Although reactivity in metalloaggregates has been addressed with respect to other processes such as the complexation of oxygen, photochemical and Diels Alder reactions (13), most studies from several laboratories, including ours, were devoted to the hydrolytic reactions. Hydroxy or oxime functionalized ligand such as 3-6, or also devoid of any function such as 7, were investigated and rather impressive results were obtained. On employing lipophilic pyridine-based ligands 3-5 $\left(\mathrm{R}=n-\mathrm{C}_{16} \mathrm{H}_{33}\right)$ complexed with $\mathrm{Cu}(\mathrm{II})$,<smiles>[R]NCc1cccc(C([R])O)n1</smiles><smiles>[R]C(O)C([R])N([R])Cc1ccccn1</smiles>

4<smiles>[R]NC([R])CNC([R7])CO</smiles>

5<smiles>[R]/C(=N/O)c1ccccn1</smiles>

6<smiles>[R]N(C)CCN(C)C</smiles>

7

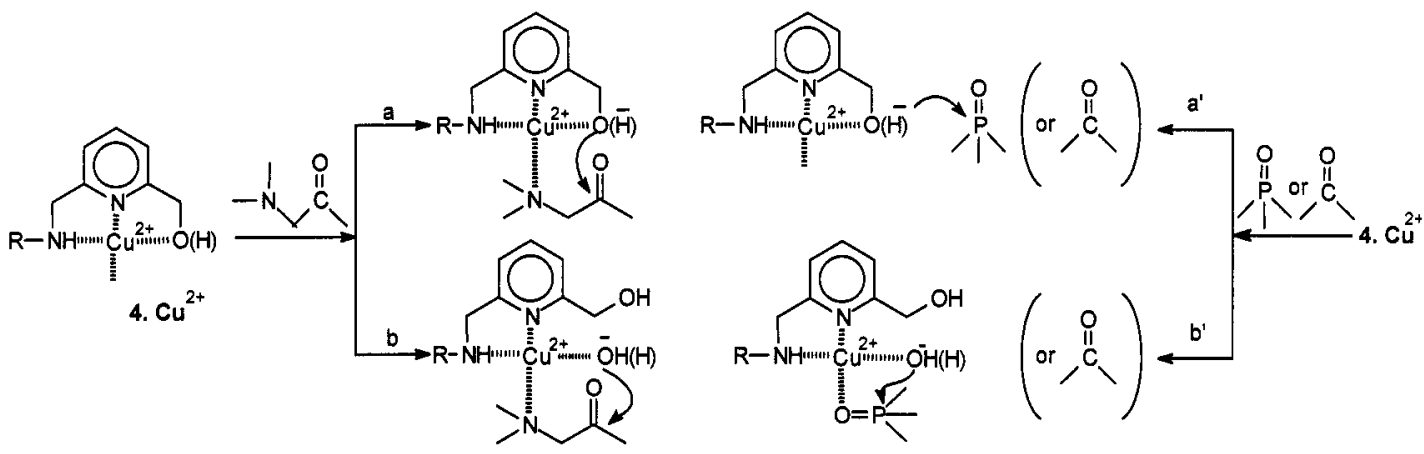

Scheme 3

$\alpha$-aminoesters were the substrates of choice for large accelerations (over a million-fold relative to buffer) and the main mode of reaction indicated in Fig.1 as path a was found to involve: (i) formation of a ternary complex (ligand/metal ion/substrate); (ii) pseudo-intramolecular attack of the activated hydroxyl on the $\mathrm{C}=\mathrm{O}$ of the ester resulting in its acylation; (iii) metal ion mediated hydrolysis of the intermediate (12d-e). Starting from the ternary complex, there may be competition between the mode of action a oulined above and that indicated as $\mathbf{b}$ involving nucleophilic attack by a coordinated water molecule. The available evidence indicates that mechanism $\mathbf{a}$ is favored in aggregates over $\mathbf{b}$, which prevails outside them in bulk 
water. The cleavage path following mode $\mathbf{a}$ is faster than $\mathbf{b}$, although the latter does not involve cleavage of any intermediate and possibly slow turnover of the catalytic system. The accelerations of six orders of magnitude cited above, impressive as they appear, are to be compared with those of the sole metal ion (3-4 orders of magnitude) and so the kinetic benefits are to be scaled down. However, non micellized analogous complexes are often less effective than the uncomplexed ion by one or two orders of magnitude so that the purely micellar advantage may be of three to four orders of magnitude in terms of reactivity and of remarkable enantioselectivity effects (see infra, Fig. 3). Some metalloaggregates are also reactive towards non-coordinating substrates: comicelles of $\mathrm{CTABr}$ and lipophilic complexes 3 with $\mathrm{Cu}(\mathrm{II})$ are very effective in the cleavage of carboxylic or phosphoric acid esters through the alternative reaction paths indicated as a' or b' (Scheme 3), the mechanistic analogs of $\mathbf{a}$ and $\mathbf{b}$, involving the coordinated ligand's function or a water molecules respectively (14). Acting through a path equivalent to $b^{\prime}$, aggregates of 6 ( $R$ $=n-\mathrm{C}_{16} \mathrm{H}_{33}$ ) complexed with $\mathrm{Cu}(\mathrm{II})$ proved to be very effective in the cleavage of phosphoric acid esters and analogous substrates (rate enhancements of over five orders of magnitude with turnover behavior), and are hence promising agents for decontamination from phosphorylated toxic materials $(15,16)$. A special case recently investigated (17) is that of the oxime functionalized ligand 6 complexed with $\mathrm{Ni}$ (II) in comicelles of CTABr. Its reactivity in the cleavage of carboxylic acid esters is remarkably unaffected by $\mathrm{pH}$ in the range 3.5-8. At $\mathrm{pH}=4$, rate enhancements of five to six orders of magnitude were observed. The reaction mechanism is suggested to involve a 2:1 [ligand:Ni(II)] species in the conformation indicated as $(5)_{2} \cdot \mathrm{Ni}^{2+}$ in Fig. 1 which may react with activated alkanoates in the small micellar volume. Surprisingly, the same complex is virtually inert toward phosphate ester.

As a matter of fact, in spite of the many studies so far published, metalloaggregates are characterized by large degree of unpredictability regarding substrate specificity, mechanistic aspects and selectivity. However, in the authenticated cases of metallocomplexes reacting through the same mechanism in aggregates and in water, and when the proper parameters affecting their reactivity can be evaluated, application of the pseudophase model to the kinetics of reactions in metallomicelles or metallovesicles indicates that the second order rate constants, $\mathrm{k}_{2 \mathrm{M}}$, are very close to those in water, $\mathrm{k}_{2 \mathrm{w}}$. Again, once concentration effects and possible changes in acid-base and metal ion binding equilibria are taken into account, no special kinetic benefit or medium effect of the aggregate can apparently be claimed (18).

\section{Selectivity}

Alteration of reaction paths and control of stereochemistry induced by reagent organization and different microenvironment was sought for and realized in a number of cases. Molecular organization in micelles or vesicles have been exploited in photochemical energy tranfer or energy conversion processes as well as in photochemical energy conversion (19). The scheme of Fig. 2 provides just an example of alteration of reaction paths occurring in aqueous solutions of metalloaggregates, as defined on the basis of mechanistic studies. Such changes could appear of little significance since they do not lead to different product distribution; yet, they have a rather remarkable effect in the stereoselectivity of $\alpha$-aminoester cleavage when chiral reagents are employed.

\section{Enantioselectivity.}

Since the early studies on reactivity in aggregates, considerable attention was devoted to the enantioselectivity issue. Moss (20) pioneered this field by investigating the deamination of chiral primary amines and finding a dramatic change from net inversion to extensive retention of configuration in going from non-assembled to micellar systems. A scrutiny of published studies, using enantiomeric substrates and chiral surfactants, indicates that enantioselectivity effects are: (i) small, if any, on employing aggregates of inert chiral surfactants; (ii) sometimes sizable but generally not impressive in the case of functionalized aggregates (see below however). In such cases, the effects due to diasteromeric interactions in aggregates, mainly at the transition state level, are virtually the same as experienced by the reacting system in bulk water; (iii) often quite remarkable in the case of chiral metalloaggregates although restriced to specific substrates such as the $\alpha$-amino acid derivatives.

As a striking exception to point (ii), very high enantioselectivities up to rate ratios of $60-70$ were reported by Moss and coworkers (21) in the cleavage of the enantiomers of $\mathrm{N}$-dodecanoyl phenylalanine in aggregates made of the (diasteromeric) tripeptide Z-L-Phe-L-His-L-Leu, CTABr, and $\left(\mathrm{C}_{14} \mathrm{H}_{29}\right)_{2} \mathrm{~N}^{+}\left(\mathrm{CH}_{3}\right)_{2}$, 
dubbed $2 \mathrm{C}_{14}$. On increasing the fraction of $2 \mathrm{C}_{14}$ up to 30 mole $\%$ of the total aggregate content, precipitation occurs; further addition to more than a $60 \%$ fraction leads to clear solutions. A very sharp increase of enantioselectivity was observed for solutions approaching from either side the heterogeneous interval. The rationale was that the highly discriminating aggregate enforces a very ordered arrangement of the reactants. As a general indication these data would point towards a highly structured aggregate with a limited particle diffusivity as a medium for large enantioselectivity effects.

The effects in metallomicelles only partly confirm the above generalization: on the one hand, the effects observed in vesicles decrease on incresing the temperature in the range of the phase transition interval as the structure of the vesicles move from a more ordered to a looser one; on the other hand, the enantioselectivities recorded for the same complex and substrate do not change remarkably on moving from a micellar matrix to the much more ordered vesicular one (22). As previously mentioned most of our studies were addressed to $\mathrm{Cu}(\mathrm{II})$ complexes of chiral ligands 3,4 (R'or R" $\neq \mathrm{H}$ ), 5 (R'or/and $\mathrm{R}^{\prime \prime} \neq: \mathrm{H}$ ) employing the enantiomeric $p$-nitrophenyl esters of amino acids such as phenylalanine (PhePNP), phenylglycine, and leucine as substrates. The rate ratios were mostly in the range 10-30 (23), and over 50 only under very special conditions (24). The very complex metalloaggregate systems and the still limited number of systems investigated do not allow extended and reliable generalizations. However, reactivity and enantioselectivity are much dependent on the presence of aggregates. As cleanly illustrated in the rateconcentration profile of Fig. 1, the rate of cleavage of the two enantiomers of PhePNP increases to saturation on increasing the concentration of the micellar 1:1 mixture of ligand $4 a\left(R=\mathrm{C}_{16} \mathrm{H}_{33}, \mathrm{R}^{\prime}=\mathrm{CH}_{3}\right.$,
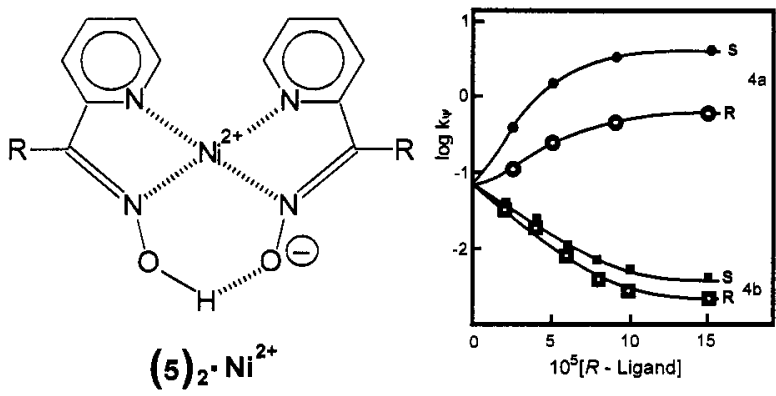
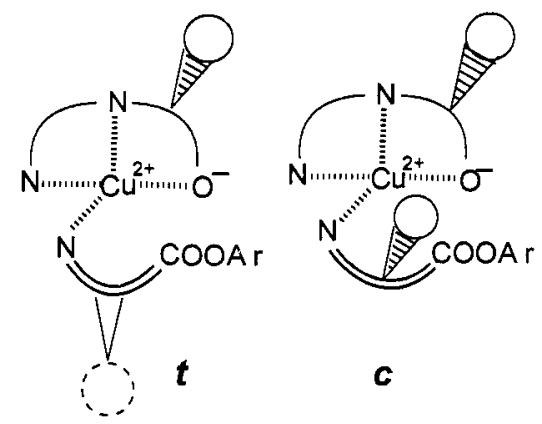

Fig. 1

$\mathrm{R}$ " $=\mathrm{H}$ ) and $\mathrm{Cu}(\mathrm{II})$, whereas a decrease is observed upon addition of the water soluble $4 \mathrm{~b}$ ( $\mathrm{R}$ and $\mathrm{R}^{\prime}=$ $\mathrm{CH}_{3}, \mathrm{R}$ " $\left.=\mathrm{H}\right)$ and $\mathrm{Cu}(\mathrm{II})$ as a consequence of the different reaction modes defined as $\mathbf{a}$ and $\mathbf{b}$ prevailing in aggregate and in water, respectively. Mode a involves the attack of the metal bound $\mathrm{OH}$ function and the chiral center of the ligand is kept closer to the reaction center so that the enantioselection results are higher (by a factor of ca. 10) than in the case of mode $\mathbf{b}$ where the ligand's chiral arm is displaced by the (activated) water molecule which assumes the role of a nucleophile. A more significant point is the fact that a statististical survey of almost two dozen cases involving aggregated ligands 3-5 indicates the following common feature. Assuming a square planar (or distorted planar) arrangement around the $\mathrm{Cu}$ (II) ion, the four strong coordination positions being taken by the two nitrogen and the hydroxyl of the ligand and by the amino nitrogen of the substrate, the complex with such configuration of the chiral carbons as to place the R, R' substituents in the "trans" like conformation react faster when they are in the "cis" type one (see Fig. 1, $t$ and $c$ ). The effects are confirmed by employing diastereomeric ligands with two chiral centers of type $\mathbf{5}\left(\mathrm{R}^{\prime}\right.$ and $\left.\mathrm{R}^{\prime \prime} \neq \mathrm{H}\right)$ : the all cis conformation is remarkably slower that any other diastereomeric geometry. The fast reacting "trans" arrangements may be either more stable or more hydrophobic than the cis one. Evidence has been obtained against the idea that steric hindrance may play a major role. The current hypothesis (22-24), although based on still scarce and indirect support, is that the cis arrangements are more hydrophilic than their diasteromeric counterparts and they react in a relatively more hydrated regions of the aggregates where the competition of mode $\mathbf{b}$ (slow) with mode a (fast) is stronger: the resulting effect is a reactiviy gap that leads to the observed enantioselectivities. 


\section{Acknowledgments.}

The author thanks his coworkers, whose names appear in the references, for their intellectual contributions. This work has been supported over the years by MURST, the Italian Ministry of University, and recently by funds of the Supramolecular Devices Project.

\section{References}

1. (a) J. W. MacBain and H.E. Martin, J. Chem. Soc. 105, 957 (1914). (b) G. S. Hartley, Trans. Farad. Soc., 31, 31 (1935).

2. Selected references: (a) J. H. Fendler, Membrane Mimetic Chemistry, Wiley: New York (1982). (b) C. A. Bunton and G. Savelli, Adv. Phys.Org. Chem., 22, 213 (1986). (c) D. D. Lasic, Liposomes, Elsevier, Amsterdam, (1993). (d) F. M. Menger, Angew. Chem. Int. Ed. Engl, 30, 1086, (1991).

3. J.-M. Lehn, Angew. Chem. Int. Ed. Engl. 27, 89 (1988)

4. (a) F. M. Menger, Accounts Chem. Res, 12, 111 (1979). (b) N. M. van Os, J. R. Haak, L. A. M. Rupert, Physico-chemical Properties of Selected Anionic, Cationic, and Nonionic Surfactacts, Elsevier, Amsterdam, (1993). (c) W. Blokzijl and J. B. F. N. Engberts, J. Amer. Chem. Soc., 114, 5440 (1992)

5. (a) F. M. Menger and C. E. Portnoy, J. Amer. Chem. Soc., 89, 4698, (1967); (b) K. Y. Yatsimirski, K. Martinek, and I. Berezin, Tetrahedron, 27, 2855 (1971). (c) L. S. Romsted in Micellization, Solubilization, and Microemulsions, K. Mittal, ed., vol. 2, p. 509, Plenum Press, New York, (1977);

6. (a) C. A. Bunton, F. Nome, F. H. Quina, L. S. Romsted, Accounts Chem. Res., 24, 237 (1991) (b) C. A. Bunton, Catal. Sci. Rev. Engl.,20, 1 (!979)

7. (a) T. C.Bruice, J. Katzhendler, and L. R. Fedor, J. Amer. Chem. Soc, 90, 1333 (1968). (b) C. Gitler and A. Ochoa-Solano J. Amer. Chem. Soc, 90, 5004 (1968). (c) R. A. Moss, R. C. Nahas, S. Ramaswani, and W. J. Sanders, Tetrahedron Letters, 4435 (1975). (d) C. A. Bunton and S. Diaz, J. Org. Chem.,41, 33 (1976). (e) S. Shinkai, N. Nakashima, and T. Kunitake, J. Amer. Chem. Soc, 100, 5887 (1978). (e) U. Tonellato in Solution Chemistry of Surfactants, K.Mittal ed., vol. 2, p. 541, Plenum, New York (1979). (f) U. Tonellato, Colloids and Surfaces, 35, 121 (1989), (g) L. Anoardi, R. Fornasier, and U. Tonellato, J. C. S. Perkin II, 260 (1981), and references therein.

8. U. Tonellato, J. C. S. Perkin II, 821 (1977).

9. R. Fornasier and U. Tonellato, J. C. S. Faraday I, 76, 1301 (1980)

10. F. M. Menger, L.G. Whitesell, J. Amer. Chem. Soc, 107, 707 (1985)

11. R. A. Moss, R. C. Nahas, S. Ramaswani, J. Amer. Chem. Soc, 99, 627 (1977)

12. L. L.Melhado and C. D. Gutsche J. Amer. Chem. Soc, 100, 1850 (1978). (b) W. Tagaki, K. Ogino, Top. Curr. Chem., 128, 144 (1985). J. Amer. Chem. Soc., 111, 5086 (1987). (d) R. Fornasier, D. Milani, P. Scrimin, and U. Tonellato, Gazz. Chim. Ital., 116,55 (1986). (e) R. Fornasier, P. Scrimin, P. Tecilla, and U. Tonellato J. Amer. Chem. Soc.,111, 224 (1989). (f) P. Scrimin, P. Tecilla and U. Tonellato in Organic Reactivity and Biological Aspects, B. T. Golding, R. G. Griffin, a.nd H. Maskill eds., The Royal Society of Chemistry, p. 223 (1995). (g) J. G. J. Weijnen, A. Koudijs, J. F. J. Engbersen, J. C. S. Perkin II, 829 (1992) and J. Org. Chem., 57, 7259 (1992).

13. R. Humphrey-Baker, Y. Moroy, M. Graetzel, E. Pelizzetti., and P. Tundo, J. Amer. Chem. Soc., 1097247 (1980). S. Otto, PhD Thesis, University of Groningen, (1998).

14. P. Scrimin, P. Tecilla, and U. Tonellato, J. Org. Chem., 56, 161 (1991)

15. F. M. Menger, E. Gan, E. Johnson, and D.H. Durst, J. Amer. Chem. Soc. 109, 2800 (1987).

16. C. A. Bunton, P. Scrimin, and P. Tecilla, J. Amer. Chem. Soc., 116, 11614 (1994).

17. (a) J. Budka, F. Hampl, F. Liska, P. Scrimin, P. Tecilla and U. Tonellato, J. Mol. Catal. A, 104, L201 (1996).

(b) F. Hampl, F. Liska, F. Mancin, P. Scrimin, P. Tecilla and U. Tonellato, submitted.

18. P. Scrimin, P. Tecilla, and U. Tonellato, and C. A. Bunton, Colloids and Surfaces, in press.

19. J. H. Fendler, J. Phys. Chem. 84, 1485 (1980)

20. R. A. Moss, C. J. Talkawski, C. J. Reger, and C. E. Powell, J. Amer. Chem. Soc., 95, 5125 (1973).

21. R. Ueoka, R. A. Moss, S. Swarup, Y. Matsumoto, G. Strauss, and Y. Murakami, J. Amer. Chem. Soc., 107, 218 (1985).

22. M. C. Cleij, P. Scrimin, P. Tecilla, and U. Tonellato, Langmuir, 12, 2956 (1996).

23. P. Scrimin, P. Tecilla, and U. Tonellato, J. Org. Chem. 58, 3025 (1993) and Tetrahedron, 51, 527 (1995). (b) M. C. Cleij, P. Scrimin, P. Tecilla, and U. Tonellato, Tetrahedron, 53, 357 (1997).

24. F. Bertoncin, F. Mancin, P. Scrimin, P. Tecilla, and U. Tonellato, Langmuir, 14, 975 (1998). 